\title{
El DifERIMIENTO DEL IMPUESTO A LAS GANANCIAS
}

\section{THE DEFERRAL OF INCOME TAX}

Hugo Ricardo Arreghini ${ }^{1}$

Fecha de recepción: 06/05/2019

Fecha de aceptación: 13/08/2019

\section{Resumen}

La explicación del resultado fiscal, aunque está fundamentada en información procedente de la normativa contable vigente, hace una interpretación particular de los criterios que esas reglas adoptan. De tal modo, es natural que se observen diferencias en el cálculo del impuesto a la renta, según se haga con una u otra metodología. Sin embargo, la supremacía en la aplicación del criterio fiscal que el contribuyente debe aceptar, le exige la adopción de un especial entendimiento, cuya manifestación tácita de acatamiento ha sido dada en función de la protección que la persona jurídica -física o ideal- recibe del Estado que la reconoce. Esta circunstancia es decisiva para comprender que el impuesto solo se define por la forma en que el Estado percibe el beneficio patrimonial que genera dicho impuesto. Por tal motivo, cualquier otra forma conceptual que permita originar diferimientos y consecuentes resultados, activos o pasivos, no tendría sentido.

Pal abras clave: persona jurídica, ente económico, activo diferido, pasivo diferido, beneficio económico participado.

1 Universidad del Centro de la Provincia de Buenos Aires (UNICEN), Facultad de Ciencias Económicas, Profesor Emérito; Universidad de Buenos Aires (UBA), Facultad de Ciencias Económicas, Profesor Consulto. E-mail: harreghini@gmail.com 


\section{Abstract}

Despite being based on current accounting regulations, the explanation of the tax result, makes a particular interpretation of the criteria adopted in these rules. Thus, it is natural to find differences in the calculation of income tax, depending on the methodology used. However, the preeminent application of the tax criterion that the taxpayer must accept requires special understanding as tacit compliance relies on the protection that the legal person, either natural or artificial, receives from the State. This circumstance is decisive to understand that the tax is only defined by how the State perceives the financial benefit giving rise to such tax. Therefore, any other conceptual form allowing deferrals and resulting assets or liabilities makes no sense.

Keyw ords: legal person, economic entity, deferred asset, deferred liability, shared economic benefit.

JEL: H22, H24, H25, M41. 


\section{Introducción}

El tratamiento del impuesto a la renta, que se manifiesta en las normas contables de forma generalizada, ofrece dificultades para catalogarlo conceptualmente dentro de los fundamentos de la Contabilidad y para efectuar su complicada aplicación en las entidades económicas pequeñas y medianas. Tales circunstancias justifican un análisis profundo de las razones que podrían motivar su aprobación.

La corriente que apunta convencionalmente a reconocerle vigencia, ha favorecido a que la aceptación de las reglas dictadas con el auspicio de los organismos profesionales, a pesar de los inconvenientes señalados, se impongan sin mayor discusión y se otorgue a su existencia una convalidación que parece definitiva.

En las casas de altos estudios, la atención de este problema se ha centrado especialmente en el análisis pragmático de los procedimientos para la puesta en funcionamiento, sin tener en cuenta sus basamentos radicales. Este escrito intenta demostrar que esa omisión no solo se debe corregir, sino que merece un estudio más severo desde la Universidad, en tanto órgano competente para consagrar su uso.

\section{Acerca del resultado de ejercicio}

Antes de hacer el análisis particular que atañe a este escrito, es pertinente realizar el correspondiente al tratamiento del resultado de ejercicio, determinante del gravamen. El resultado es, siempre, la consecuencia de una confrontación, pero los efectos del enfrentamiento natural que ella requiere pueden estar sujetos a diferentes valoraciones que los ubiquen en una escala donde prevalezcan, desde los extremos que califican el valor, tal como se entiende por la cualidad o por la efectividad que lo expresa.

Ambas posiciones no se manifiestan demasiado alejadas cuando se intenta definir aquello que las representa, puesto que los atributos que caracterizan su significado parecen conducir a apreciaciones similares a los estándares de la realidad, tan difícil de revelar. Ortega y Gasset (1961) explica que:

La realidad, pues, se ofrece en perspectivas individuales. Lo que para uno está en último plano, se halla para otro en primer plano. El paisaje ordena sus tamaños y sus distancias de acuerdo a nuestra retina, y 
nuestro corazón reparte los acentos. La perspectiva visual y la intelectual se complican con la perspectiva de la valoración. (p. 20)

Aun así entendida, debiera considerarse más objetivamente aquello que asegure ser la mejor calificación de sus condiciones naturales, procurar los dividendos más cuantiosos por la posesión de la mayor inversión, o bien reputarse como más rico por gozar de recursos más potentes.

En cualquiera de los supuestos indicados, la respuesta está señalada por la lógica, que solo parece desmentir una deficiente administración al verificarse la real utilización de los supuestos activos empleados, la ocurrencia de un inesperado hecho que cambiara la racionalidad de los acontecimientos vinculados, o la intervención desproporcionada de una fuerte carga emocional que inclinara a tomar decisiones inesperadas.

$\mathrm{Ni}$ siquiera la pertenencia mejor sostenida asegura que "lo que viene a propósito", por ser pertinente, conduce inexorablemente a la mejor consecuencia. Esto significa que la acción sobre los elementos involucrados solo es verificable cuando ha sido ejecutada pero, además, cuando el significado que se le atribuye demuestra haber sido consentido.

Cuando la perspectiva de aplicación de algún bien se haga posible, ello no será en sí mismo, garante de una efectiva utilización. Al ser un recurso, no haber dispuesto su empleo demostrará ser insuficiente para hacer la apreciación de los potenciales efectos a los que se podría aspirar con él.

Lo expuesto permite confirmar que la determinación de resultados exige la existencia del hecho que lo genera. No alcanza con la tenencia del patrimonio para atribuirle una acción útil que no se haya asegurado con la ejecución del acto que lo incluye y da validez a su uso.

La convalidación de esa manifestación requiere correspondencia con espacios que puedan liberarse de circunstancias coyunturales y superar la influencia estacional y, también, no ser afectada por cualquier incidencia de factores desequilibrantes de la decisión para convertirla en arbitraria o excepcional. Exige, además, ampararse en módulos que mantengan su condición para valorar y medir adecuadamente.

\section{El Resultado Entendido Con La Teoría De La Utilidad}

La convención expresada ha sido menguada por los últimos pronunciamientos profesionales, proclives a dar a la contabilidad un objeto que consideran más 
útil, comprometido con la explicación de los beneficios que las organizaciones pueden obtener de los actos que las incluyen dentro de su ámbito de desarrollo, aunque no tengan en ellos una participación directa.

Esa concepción da preeminencia a la contemplación evolutiva de la valoración de sus patrimonios netos, para interpretar la variación periódica potencial de las unidades analizadas. Además, se funda en que el fin perseguido por la actividad económica, que intenta acrecentar los medios para satisfacción de necesidades de quienes la financian, se consigue con el beneficio estimable, al comparar lo que el medio permite convalidar con la observación de los valores razonables que se les puede asignar.

Esa presuntiva estimación comprende el "efecto de las interacciones entre la empresa y el ambiente económico, legal, social, político y físico" (García y Werbin, p.979). Le da, por ello, un carácter amplio inclusivo de aspectos que no exigen integrarse con lo perteneciente, y admiten la incursión hecha para la apreciación de los activos y pasivos de supuestos ingresos netos futuros y de valoraciones tomadas de otras fuentes del entorno que considera asimilables.

La disposición de un beneficio así computado promueve, de este modo, cierta perplejidad con respecto a las garantías que ofrece tal procedimiento, que se sustenta en la revaloración de los componentes del patrimonio neto para hacer el cotejo de su significado en dos momentos consecutivos y determinar la magnitud anual de su crecimiento.

Estas reflexiones se realizan para recordar que la determinación que da fundamento al gravamen exige certeza sobre los hechos determinantes y sus efectos, y que este modo de entender la concreción del resultado periódico como un beneficio calculado en base al cotejo de magnitudes surgidas del activo y pasivo de los balances de dos ejercicios, en cierto modo ha contribuido a legitimar el método de diferimiento del impuesto a las ganancias en base a cambios patrimoniales y descarte de la aplicación procedente de los ingresos y costos del mismo lapso. De alguna manera, este cambio metodológico incide en las conclusiones que podrán obtenerse al cabo de este análisis.

Aun se podría conjeturar si es equivocada la denominación que en nuestro país se ha dado al gravamen, ya que no necesariamente debería entenderse como significativa del objeto que persigue. Puede observarse que, en el cálculo de la base imponible, sigue prevaleciendo el concepto de ganancia definido con ingresos y gastos, en abierta contradicción con la teoría de que el beneficio de la empresa debe determinarse haciendo el cómputo del incremento en la valoración comparada, operada en el patrimonio neto al cabo del período anual 


\section{Diferimiento del impuesto sobre el resultado}

Tomar partido por esta preferencia parece estar confirmado por la indiscutible posición de que el impuesto a las ganancias no está integrado al resultado porque es su consecuencia, ya que la que recibe el impacto de la carga fiscal, en este caso, es la entidad jurídica y no los efectos económicos de su actividad..

No obstante ser obvio, nos atrevemos a aclarar que la contabilidad - al procesar la información para luego exponer, por lo menos, la situación patrimonial y los resultados obtenidos (y las variaciones ya aludidas) - no se propone como objetivos (1) ni la determinación de montos sujetos a impuestos, (2) ni el cálculo de las ganancias disponibles para ser distribuidas (por ejemplo, en forma de dividendos). Tales informaciones surgirán como subproductos, teniendo en cuenta los datos suministrados por la Contabilidad....Las mediciones deberían ser independientes de los problemas del dividendo y del impuesto, pero al mismo tiempo, deberían facilitar la solución de esos problemas como también de otros relativos a la posición financiera y los resultados de la operación. (Bértora, 1975, pp. 60-61)

La decisión con respecto a la disposición del resultado es responsabilidad de la entidad jurídica, nunca de la empresa -ente económico- que la origina como un instrumento de la actividad emprendida con ese objetivo. Este compromiso, aunque tácito, está vigente desde el mismo momento en que el Estado reconoce la existencia natural o ideal de la persona, y acuerda protegerla con derecho a reclamarle recursos compensatorios de esa acción.

La justificación para hacer incidir efectos temporarios que se deduzcan de las diferencias que presenta el tratamiento contable con respecto al impositivo, tiene un fundamento único que se refleja en la intención de procurar el adecuado devengo de la ganancia contable periódica, asumiendo que el cálculo del gravamen emplea un modo distinto de computar en el tiempo, la ubicación de algunos ingresos y gastos, o de sus contravalores.

La particular concepción que procura evitar, en este caso, la violación del devengo, intenta corregir la posición temporaria que el fisco impone para la declaración jurada del impuesto, a través de su adecuación al enfoque sostenido por las normas contables. En la síntesis que proponen esos mecanismos de ajuste, se observa la intención de dar a los formadores del resultado un sentido contable que se presenta temporalmente de modo diverso en la ley, 
considerando que, si se siguieran las convenciones sostenidas por la profesión contable, no respetaría la ubicación que les corresponde. Se trata de un modo de actuar que se compadece más de cerca con un devengo acorde a la idea que vincula el ingreso neto - diferencial - con la carga que atribuye ser el gravamen, pero al que se debe ocultar reminiscencias que lo vinculen con el cuadro de ganancias y pérdidas. Al respecto, Oklander (1983) dice:

Incluimos en este vastísimo abanico de 'aplicaciones' de la Contabilidad, a la administración de la gestión empresaria en su faz operativa, al margen del nivel de la 'toma de decisiones`. Lo mismo podemos decir de la contabilidad como acto jurídico vinculante' y, dentro de este campo, como base para la determinación tributaria" (p. 931)

Para mostrarse respetuoso de esa consigna utilitaria que impone el criterio de ser fiel a la mejor definición que pretende el mercado, la corrección obligada debería afectar un activo o un pasivo que determine un impuesto contable menor que el impositivo o viceversa, en lugar de imputarse como consecuencia de su deducción por observación de los ingresos y gastos que pudieron haber originado esas diferencias.

El origen que a ello se atribuye conduce a una interpretación distinta de sus fundamentos. Cuando proviene de divergencias basadas en los componentes del estado de resultados, estima el gravamen como un gasto relativo al período en el cual es reconocido el beneficio. Esta diferencia se denomina impuesto a las ganancias diferido y debe revertirse en el futuro. En cambio, si procede de los rubros del balance, se considera como una ventaja que disminuirá el pago futuro o como una obligación que será satisfecha más adelante.

De cualquier modo, la imputación formal permanece respetuosa de un tratamiento que disminuye el beneficio generado por la actividad que lo provoca, debido a que se sigue considerando el gravamen como un factor determinante de ese incremento patrimonial restante, que no lo excluye del estado que expresa los resultados periódicos. Se sigue manifestando dentro del cuadro de ganancias y pérdidas, de modo que el resultado distingue magnitudes diferentes como anteriores o posteriores al impuesto.

La situación, según la declaración jurada, se contabilizaría como:

Impuesto a las ganancias

a Impuestos a pagar
100

100 
$\mathrm{Al}$ computarse el diferimiento con un beneficio contable menor al impositivo, se registrará como:

$$
\begin{array}{ccc}
\text { Impuesto a las ganancias } & 95 \\
\text { Activo por impuesto diferido } & 5 & 100 \\
\text { a Impuestos a Pagar } & & \\
\text { O, con un beneficio contable mayor al impositivo, como: }
\end{array}
$$

$\begin{array}{crr}\text { Impuesto a las ganancias } & 105 & \\ \text { a Impuestos a Pagar } & 100 \\ \text { a Pasivo por impuesto diferido } & 5\end{array}$

En uno u otro proceder, se verificará una modificación del gravamen imputable, que alterará el resultado antes de impuestos para llegar al resultado después de impuestos a considerar como expectativa de distribución de utilidades a los socios. Con respecto a la distribución de los beneficios de las sociedades, Mathews (1947) dice:

Se supone implícitamente que el impuesto a pagar representa una transferencia de beneficios más bien que un gasto con cargo a los mismos en la cuenta de pérdidas y ganancias. La razón principal de este supuesto es que el importe del impuesto a pagar no puede ser calculado hasta que se conoce la cuantía de los beneficios propiamente dichos. (p.214)

\section{Persona jurídica y entidad económica}

Un necesario salto hacia lo disponible por el ente jurídico, que muestra con bastante claridad esa necesaria discriminación y los caracteriza como destinados a diferentes entidades, podría representarse como la ecuación siguiente: Resultado económico obtenido por la empresa - Resultado para el gobierno del Estado = Resultado para el propietario. Es decir, "el Fisco, a través del impuesto a la renta, es un socio que, indudablemente, participa en los resultados de la explotación (Biondi, 1973, p. 104).

Para dar mayor fuerza al razonamiento que justifica esta observación y fortalecer la negativa categorización del impuesto como generador del resultado, 
en vez de una consecuencia del mismo, se puede acudir a las disposiciones del nuevo Código Civil y Comercial de la República Argentina (2014) y a los argumentos que las respaldan. Ello permite entender la ubicación de la persona jurídica que tiene como ejecutora a la empresa que ha creado y recibe el producto de su acción, después de haberlo participado con el gobierno del Estado.

El artículo 141 es esencial para inferir la amplitud conceptual que caracteriza a la persona jurídica, que coloca su figura por encima del objeto específico que le permitirá cumplir su función, como la actividad económica, entre otras. Conduce a interpretar que la sociedad comercial es, entonces, la reunión de personas que se agrupan para gestionar una empresa y cumplir con un proyecto cuyo resultado permitirá a los integrantes de la impulsora - la persona jurídica -, conseguir un beneficio que deberá compartir, de algún modo, con el Estado que le obliga a pagar un impuesto basado en ese acrecentamiento obtenido en el capital empleado.

El Código facilita la comprensión de lo anterior, ya que permite interpretar la perspectiva de que una persona jurídica - en este caso una sociedad anónima - pueda crear más de una unidad identificable que realiza actividades económicas y está constituida por combinaciones de recursos humanos materiales y financieros conducidos y administrados por un único centro que toma decisiones encaminadas al cumplimiento de los fines específicos para los que fue creado. La personalidad jurídica es escindible de lo económico, que puede ser el fin de la constitución de aquella y este no la califica, y es, a su vez, independiente de la persona de sus accionistas, propietarios o participantes.

Entender esta forma de relacionar el ejercicio de la propiedad con distintos objetos a los que puede ser comprometido (algunos de ellos con un significado trascendente para su desenvolvimiento y resultados obtenidos) ha contribuido a la consagración reciente, en nuestro derecho, de las sociedades unipersonales que habían sido ignoradas hasta el advenimiento del nuevo Código. Resulta así más clara la posibilidad de que el sujeto cumpla actividades no relacionadas, pero igualmente importantes, para ser consideradas con valoración separable, ya que, ahora, pueden ser tratadas sin problema como objeto de una sociedad anónima, es decir, de un sujeto con una personalidad a la que se reconocen derechos y obligaciones respondiendo a ese fin particular.

En rigor, esto que se puede ver reforzado por el hecho comentado, tiene un respaldo indiscutible por la lógica en que se sostiene. De modo que se constituye en uno de los argumentos más fuertes para negar que el compromiso que asume la persona jurídica, en su tributación del impuesto a las ganancias, pueda tener otra comprobación que la que derive de la actividad económica 
efectuada y ello permita asignarlo no como su causa, sino como un efecto de haberla desarrollado.

El impuesto puede ser calificado como procedente de los beneficios que la persona es capaz de generar recurriendo a objetos diversos. Esto es más visible cuando se trata del que se aplica a las personas físicas afectadas, según tengan ingresos provenientes de fuentes separadas, que la ley se ocupa de considerar con diferente peso en categorías diversas. Es posible que, cuando de ello se trata, apele a determinar el ingreso utilizando técnicas distintas para su determinación - devengo o percepción efectiva -. Comúnmente, formula tratamientos que responden a la situación que provoca al sujeto imponible las cargas sociales que le ocasiona la familia.

La diversidad de orígenes y aplicaciones que se le puede adjudicar concurren, sin embargo, a señalar que su fundamento está en el acrecentamiento patrimonial que atribuye la ley a los ingresos netos de gastos que la persona obtiene anualmente y privilegia la observación de que el gravamen no tenga perspectiva de trasladarse a otras entidades o imputarse a otros períodos, lo que hace menos posible su flexibilización o reconversión.

Es por ello elemental que la naturaleza del tributo - con su discutible denominación local - defina su base imponible con total independencia del resultado o del acrecentamiento patrimonial que ofrecen los estados contables, que solo le sirven como instructivo orientador, y el contribuyente ha aceptado y confirma acatar al declarar juradamente que está de acuerdo con ello.

La ubicación, conforme a tal consentimiento, no admite otro lugar que su correspondencia con el año atribuido a la exacción de algún modo pactada y atendida. De esta manera, su posición efectiva está regida por momentos de cumplimiento que no manejan los efectos que podrían dirigirse a desmentir probables cálculos de anticipación o postergación hechos con otra lógica - los establece unilateralmente el Fisco, han sido aceptados de antemano -.

Las especulaciones que pretenden otra incidencia temporal para el gravamen no son aceptables porque apuntan a considerar la materialización de los componentes de la base con que se lo debe calcular - que, como se ha observado, es poco clara - en normas sostenidas en la Contabilidad, pero tienen su demostración definitiva en la ley. Además, el procedimiento de atribución no está referido al sustantivo - el impuesto mismo - sino a los causantes que permiten su cálculo.

Se incurre en el error de querer interpretar el devengo del contenido - un valor neto ya homogeneizado por disposiciones legales inapelables - acudiendo al diverso criterio impuesto, en las normas contables, a sus integrantes 
comprometidos con esa mezcla. Se busca justificar el origen del total conseguido a través de su desmembramiento, para encontrar sus distintos fundamentos radicales y exigir su consideración con tal criterio, cuando se ha consentido que no es aplicable.

\section{El diferimiento en las normas contables argentinas}

Al hacer el análisis de las normas contables que tratan este asunto, se advierte la dificultad que ofrece para dar una solución a ese círculo vicioso que se instala a partir de otorgar al impuesto a las ganancias, en la Resolución Técnica (RT) 16 de la Federación Argentina de Consejos Profesionales de Ciencias Económicas (FACPCE), el carácter de integrante del resultado. A saber,

4.2 Evolución patrimonial [...] varía como consecuencia de: [...] b) el resultado de un período, que es la variación patrimonial no atribuible a las transacciones con los propietarios y que resulta de la interacción de: 1) flujos de ingresos, gastos, ganancias y pérdidas; 2) los impuestos que gravan las ganancias finales; 3) en los grupos económicos, la participación de los accionistas no controlantes (si los hubiera) sobre los resultados de las entidades controladas. [...] Son gastos las disminuciones del patrimonio neto relacionadas con los ingresos (FACPCE, 2000a).

La respuesta puede estar insinuada por la deducción a que conduce el modo de exposición adoptado en la RT 9 que acepta la comprobación de dos tipos de resultados: antes y después de impuestos, con los que parece dejar resuelta esta duda.

ANEXO - MODELO DE ESTADOS CONTABLES" de la Resolución Técnica No 9 de F.A.C.P.C.E. para el "Estado de Resultados" muestra al "Impuesto a las Ganancias" restado de la "Ganancia (Pérdida) antes del Impuesto a las Ganancias", para arribar, después de deducir el "Impuesto a las Ganancias" a la "Ganancia (Pérdida) de las operaciones", lo que sería una indicación de que el gravamen es una consecuencia y no una causa del resultado (FACPCE, 1987).

No obstante, la afirmación de que el neto es derivado de las operaciones introduce nuevamente en la duda, también impulsada por la misma RT 9 
cuando trata de precisar cómo proceder, que alude al impuesto cargado a resultados como aplicado a la ganancia o pérdida contable antes de impuesto.

C.7. Impuesto a las ganancias [...] Deben presentarse: a) una conciliación entre el impuesto cargado a resultados y el que resultaría de aplicar a la ganancia o pérdida contable (antes de impuesto) la tasa impositiva (FACPCE, 1987).

Luego de esta prolongada incursión, el lector debería, dificultosamente, incorporar la convicción de que la norma reconoce que el impuesto no participa como factor del resultado de la unidad económica, sobre todo si observa que en la misma RT 16 se afirma que el impuesto a las ganancias tiene dependencia del flujo de ingresos, gastos, ganancias y pérdidas y que el resultado neto a que se refiere no es el que corresponde al que quedaría después de haberlo restado del producido por la actividad desarrollada.

4.2.3 Impuestos sobre las ganancias. Estos impuestos afectan resultados netos, de modo que dependen de los flujos de ingresos, gastos, ganancias y pérdidas (FACPCE, 2000a).

Parece prevalecer, entonces, que la idea sustentada finalmente ha sido la de entender que existen los dos niveles, con lo que se aceptaría que hay dos tipos de resultado: el de la gestión y el que es disfrutable por la persona, tal como se ha deducido anteriormente al explicar la distinción entre entidad económica y persona jurídica.

\section{Devengo}

Ya que la cuestión está planteada, especialmente como vinculada a la ubicación de los efectos dentro de un lapso determinado, se debería observar cómo la norma contable define el tema de devengo.

En la RT 17 2.2, el devengo es tratado como el efecto reconocible en el período en que ocurren los actos o los hechos y los distingue de los que podrían determinarse como correspondientes al momento en que se produjeran los ingresos y egresos de fondos relacionados. 
Los efectos patrimoniales de las transacciones y otros hechos deben reconocerse en los períodos en que ocurren, con independencia del momento en el cual se produjeron los ingresos y egresos de fondos relacionados (FACPCE, 2000b).

Esto podría interpretarse como aplicado a cualquier concepto generador de ingresos, gastos, variaciones patrimoniales de cualquier índole, lo que validaría que se ignore la relación que entre ellas pudiera establecerse. Entendido de tal modo, permitiría desconocer uno de los supuestos básicos de la Contabilidad que, al asociar los ingresos con sus gastos, indica que es el ingreso el determinante del período en que debe reconocerse el resultado que los relaciona y los incluye.

Otra vez, esta manifestación parece contradictoria con lo que sostiene la norma contable en la RT 16 4.2.2, que al referirse a los ingresos, gastos, ganancias y pérdidas, reconoce la relación de los ingresos con sus costos.

Son gastos las disminuciones del patrimonio neto relacionadas con los ingresos. [...] Mientras que los ingresos tienen gastos (FACPCE, 2000a).

La lectura de la RT 42 (FACPCE, 2015) que indica que el impuesto a las ganancias se reconocerá sobre la base del método del diferido, enseña que está "basado en el balance" y que el mismo "requiere" que se "reconozcan las consecuencias fiscales actuales y futuras de las transacciones y otros sucesos que se hayan reconocido en los estados contables."

$\mathrm{Al}$ referirse a las diferencias temporarias, debe interpretarse que está apelando al uso del devengo, es decir, a reconocer la procedencia del "derecho" que se adquiere "a recibir una retribución por razón de trabajo, servicio u otro título", que entiende acreditados con "la determinación de los saldos al cierre del período de los activos y pasivos" contables, para establecer las diferencias temporarias que ellos presenten con respecto a los que surgen de su base fiscal. Esas diferencias multiplicadas por la tasa de impuesto permitirán, cuando sean deducibles, determinar los activos por impuesto diferido y, cuando sean imponibles, los pasivos por impuesto diferido.

El procedimiento de aplicación así establecido está señalando que su sustentación responde, en alguna medida, a argumentos contingentes impulsados por el criterio predominante de la revaloración actual hecha en función de consecuencias futuras o asimiladas del entorno, lo que transforma su determi- 
nación en presunciones que podrán o no concretarse. Tal es así, que la misma norma en 4.8 le otorga un carácter excepcional:

Consideración de hechos contingentes. Los efectos patrimoniales que pudiere ocasionar la posible concreción de un hecho futuro (no controlable por el ente emisor de los estados contables se reconocerán de acuerdo con lo expuesto a continuación: a) los favorables sólo en los casos previstos en la sección de impuestos a las ganancias. b) los desfavorables cuando: 1) deriven de una situación existente a la fecha de los estados contables; 2) la probabilidad de que tales efectos se materialicen sea alta y; 3) sea posible cuantificarlos en moneda de una manera adecuada. El activo resultante de un efecto patrimonial favorable cuya concreción sea virtualmente cierta no se considerará contingente y deberá ser reconocido (FACPCE, 2000b).

Parece indiscutible que el devengo del impuesto a las ganancias, tal como se lo impone en la norma para su diferimiento, no respeta lo que se establece en la RT 17 sección 2.2 (FACPCE, 2000b) porque deriva de hechos que ocurren en el período que indica la ley y procede de un acto aceptado que no se ha cuestionado. Mal interpretado como gasto, tampoco responde a un ingreso al que pueda imputarse claramente, sobre todo si la determinación del resultado puede considerarse, aunque sea parcialmente, la consecuencia de una comparación de valores establecidos como estimación que apeló al mercado o a la actualización de ingresos netos futuros.

Cumpliendo con los objetivos que el gobierno del Estado persigue, diferentes de los que pueden sostenerse desde la visión de los organismos profesionales con sus normas contables, la Ley del Impuesto a las Ganancias establece la forma de determinar la ganancia periódica que debe servir para el cálculo del gravamen. Lo fundamenta en argumentos que privilegian basarse en la capacidad de pago del contribuyente, que le permitan cumplir su obligación sin mayores dificultades a través del empleo de una normativa clara, indubitable, interpersonal y neutral, que respete el principio de legalidad que indica cómo y cuándo se deben cuantificar los hechos económicos a los que se refiere y, a su modo, calificar la causa eficiente del beneficio que grava y determinar su apropiación de modo que los efectos que toma en consideración, extraídos de la información que proporcionan los contribuyentes, conduzcan a una exacción periódica irreversible. 


\section{Patrimonio diferido e incertidumbre}

Los diferimientos de activos y pasivos están garantizados cuando demuestran su efectiva correspondencia con resultados de ejercicios por venir. Los activos así calificables se caracterizan por estar preparados para asumir su función en la producción de beneficios, cuando tienen condiciones para cumplir su cometido es válido aceptar que tienen capacidad para ello. Los pasivos admiten ser ingresos diferidos cuando son la derivación de prestaciones de servicios o ventas no cumplidas todavía, por los que se han recibido adelantos, y obligan a ser satisfechos en períodos próximos. Esas posiciones en el patrimonio deberían representar condiciones exentas de incertidumbre. En ambos casos, su registro exigiría que el resultado que se espera de su efectiva influencia se concretara sin inconveniente en un ejercicio futuro.

De este tipo son también los que se originan en quebrantos impositivos, sobre todo cuando están condicionados por la expectativa de la prescripción, debido a que los expone la realización de actividades que no ofrecen seguridades de recuperación de pérdidas, pero este no es el caso.

No tendría sentido reconocer un activo por impuesto diferido porque el efecto que busca justificarlo no corresponde a un bien que se utilizará en producir algo, sino que está supuestamente entendido como un costo que será gasto en el futuro y, en cambio, es una contribución que se supone no apropiable por el Estado todavía, pero se acepta pagarla hoy, como está establecido en la Ley. Tampoco se justificaría aceptar la existencia de un pasivo diferido, debido a que no corresponde a un ingreso que se recibió por anticipado, pero, como está expresado, se podría atribuir a una exacción que el contribuyente tiene ya por exigible, pero que el Estado considera gravamen futuro.

Todo encamina a considerar que lo dispuesto en el estatuto legal tiene la fuerza suficiente para dar solución indiscutible a este asunto e inhibe cualquier interpretación suspensiva o resolutoria que postergue su resolución.

\section{Calificación del impuesto}

La interpretación que recoge el registro que impulsa lo transado, refrendado por el contribuyente, también se confirma cuando se investiga alrededor del significado de los hechos y se les quiere otorgar su más adecuada acepción.

En esa búsqueda del concepto que mejor explica las consecuencias que la contabilidad debe informar, apelamos al diccionario del idioma y nos encon- 
tramos con que no se trata de un gasto que indica el efecto del consumo o de un costo para emplear el dinero en algo, sino de una erogación que es la acción para distribuir bienes o caudales. Pero esa disposición que se hace del beneficio logrado, no se ejecuta como consecuencia de haber sido discutida con la otra parte para llegar al acuerdo y, debido a ello, debe considerarse una apropiación que hace propio del Estado una cosa, ya que no puede entenderse una pérdida que supondría carencia, privación de lo que se poséa, daño o menoscabo que se recibe en al go, no tener al go que se tenía. Una relación más cercana con la naturaleza del acto que implica el pago de un impuesto de este tipo, lo define como tributar que es pagar al Estado para las cargas y atenciones públicas, como reconocimiento de la protección que otorga a las personas jurídicas.

\section{Comentarios finales}

El análisis efectuado facilita entender que existen argumentos suficientes para considerar que el impuesto a las ganancias, aplicado a cualquier persona jurídica - física o ideal -, es un gravamen directo no trasladable que:

1. Grava los beneficios que la persona obtiene de una entidad por ella creada para realizar una actividad económica.

2. La base imponible se establece con argumentos proporcionados por los estados contables que informan los resultados obtenidos por la entidad económica generadora del incremento patrimonial, pero esa información es adaptada a los fines fiscales que terminan por ser los fundamentos de la exacción a favor del Estado.

3. El gravamen al acrecentamiento patrimonial, es la participación que el Fisco obtiene en el año fiscal de los resultados que la entidad económica proporciona para ese incremento.

4. El cálculo del impuesto se efectúa siguiendo las disposiciones de la ley, de modo que su determinación conduce a establecer la cantidad que corresponde al ejercicio anual y no está afectada por otro modo de devengar la carga que produce en el patrimonio de la persona jurídica.

5. En consecuencia de la inamovilidad de ese efecto impositivo del período establecido por ley, no se justifican atribuciones distintas en carácter de activos, de pasivos o de impuestos diferidos que se basen en otro modo de devengo. 
6. En la exposición de los resultados, el estado contable que los informa concluye con expresar el que corresponde a la entidad económica, al que se deduce el tributo determinado en base a la ganancia de la unidad - la participación fiscal en ese resultado -, para explicar el neto que constituye el beneficio obtenido por la persona jurídica.

\section{Bibliografía}

Basile, D. S. (1975). Los principios de contabilidad general mente aceptados. Buenos Aires, Argentina: Ediciones Nueva Técnica y Cía. Editora Los Cardales.

Bértora, H. R. (1975). Teoría dela contabilidad, Buenos Aires, Argentina: Ediciones Macchi.

Biondi, M. (1973) Ensayos sobre teoría contable - Tratamiento de los quebrantos impositivos. Buenos Aires, Argentina: Ediciones Macchi.

Federación Argentina de Consejos Profesionales de Ciencias Económicas (1987). Resolución Técnica 9: Normas particulares de exposición contable para entes comerciales, industriales y de servicios.

Federación Argentina de Consejos Profesionales de Ciencias Económicas (2000a). Resolución Técnica 16: Marco conceptual de las normas contables profesionales distintas a las referidas en la Resolución Técnica $\mathrm{n}^{\circ} 26$.

Federación Argentina de Consejos Profesionales de Ciencias Económicas (2000b). Resolución Técnica 17: Normas contables profesionales: desarrollo de cuestiones de aplicación general.

Federación Argentina de Consejos Profesionales de Ciencias Económicas (2015). Resolución Técnica 42: Normas contables profesionales. Modificación de la Resolución Técnica $n^{\circ} 41$ para incorporar aspectos de reconocimiento y medición para entes medianos.

Fernández, L. O. (2005). El concepto de devengado en el impuesto a las ganancias. Revista Impuestos, p. 991.

García N. y Werbin, E. (2010). El concepto del beneficio contable. Enfoques activo-pasivo versus ingresos-gastos. Profesional \& Empresaria $D \& G, 132$, p. 979. Jarach, D. (1957). Curso superior de derecho tributario. Buenos Aires, Argentina: Liceo profesional CIMA.

Mathews, R. (1974). Contabilidad para economistas. Madrid, España: Ed. Aguilar S.A. 
Hugo Ricardo Arreghini

Oklander, J. (1983). Las normas legales y fiscales y los P.C.G.A. ¿Conflictos de divergencias y posible integración? Contabilidad y administración, Tomo XIII. p. 931.

Ortega y Gasset, J. (1961). El espectador. Madrid, España: Biblioteca Nueva.

(C) 2017 por los autores; licencia otorgada a la revista Escritos Contables y de Administración. Este artículo es de acceso abierto y distribuido bajo los términos y condiciones de una licencia Atribución-No Comercial 4.0 Internacional (CC BY-NC 4.0) de Creative Commons. Para ver una copia de esta licencia, visite https://creativecommons.org/licenses/by-nc/4.0/ 